\title{
Synthesis of Mesoporous Zinc Oxide without Template
}

\author{
B. Murguía-Martínez ${ }^{1}$, A. Medina ${ }^{1}$, S.E. Borjas-García ${ }^{2 *}$, V. Sayil ${ }^{1}$, P. Martínez-Torres ${ }^{2}$, L. Béjar ${ }^{3}$ \\ ${ }^{1 .}$ Instituto de Investigación en Metalurgia y Materiales, UMSNH, Morelia, Michoacán, México. \\ ${ }^{2}$ Instituto de Física y Matemáticas, UMSNH, Morelia, Michoacán, México. \\ ${ }^{3}$ Facultad de Ingeniería Mecánica, UMSNH, Morelia, Michoacán, México. \\ *Corresponding author: sborjas@ifm.umich.mx
}

Mesoporous materials have pore sizes in the range from 2 to $50 \mathrm{~nm}$. These kinds of materials can be used in applications such as catalysis and molecular sieves [1]. Zinc oxide ( $\mathrm{ZnO})$, with a binding energy of 60 meV and a wide electronic band gap of $3.37 \mathrm{eV}$, has attracted great attention to itself due to its wide range of applications such as varistors, UV absorbers, antibacterial, light emitters, dye degradation, solar cells, gas sensors, photoluminescence materials and transparent conductors [2]. Several chemical methods such as sol-gel, hydrothermal micro emulsion, homogenous self-assembly, microwave-assisted hydrothermal, spray pyrolysis, electrochemical methods and direct precipitation have been reported for the synthesis of $\mathrm{ZnO}$ nanostructures [3]. For the synthesis of mesoporous zinc oxide, the sol-gel technique has clear advantages over other techniques due to the excellent control of the composition, the homogeneity at the molecular level due to the mixture of liquid precursors and the lower temperature of crystallization, in addition to this work, a template was not used to obtain mesoporous particles. The mesoporous zinc oxide in this research work, was prepared using a hydrothermal soft treatment. In the first step, two solutions were prepared. The first one was obtained by dissolving $0.020 \mathrm{~mol}$ of zinc acetate dihydrate in $1.20 \mathrm{~mol}$ of distilled water. For the second solution, $0.02 \mathrm{~mol}$ of sodium hydroxide was dissolved in 1.20 mol of distilled water. In a second step, the Na-solution was added slowly (drop by drop) to zinc acetate solution and stirred at $500 \mathrm{rpm}$ for $30 \mathrm{~min}$ at room temperature to obtain a material with a molar ratio of Zinc: $\mathrm{NaOH}: \mathrm{H}_{2} \mathrm{O}=1: 1: 120$. The gel obtained was aged in a stainless-steel autoclave at $120^{\circ} \mathrm{C}$ for 1 day. After the hydrothermal treatment, the sample was washed with distilled water and centrifuged at $12000 \mathrm{rpm}$ for $10 \mathrm{~min}$. After that, the material was dried at $80^{\circ} \mathrm{C}$ for 1 day. Furthermore, it was necessary to calcine the sample at $560{ }^{\circ} \mathrm{C}$ for $1 \mathrm{~h}$ to eliminate the organic residues. The microstructural characterization was carried out by using a Bruker D8 X-Ray Diffractometer in order to identify the phases formed Oafter synthesis procedure. The morphology of as-synthesized sample was analyzed by using a high-resolution Scanning Electron Microscope FEG-SEM JEOL JSM 7600.

Figure 1 shows an X-ray diffraction pattern. This figure shows several peaks corresponding to $\mathrm{ZnO}$ phase which correspond to wurtzite phase with hexagonal crystal structure.

Figure 2 shows a SEM image; the size of the particles is in the range from 0.2 and $0.7 \mu \mathrm{m}$ with a hexagonal shape (figure 2a). In addition, the particle size is directly related to the ionic strength. After nucleation, the excess hydroxyl ions in the solution are adsorbed on the polar faces of the growing particles to form of hexagons shape and the water and zinc acetate induced a considerably accelerated growth [4].

On the other hand, in figure $2 \mathrm{~b}$ it is possible to observe the presence of mesopores on hexagonal crystals, the size of mesopores are less than $50 \mathrm{~nm}$. The concentration of hydroxyl ions played an important role for the shape and size of the hexagonal crystals. The nature hexagonal shape of $\mathrm{ZnO}$ could help in the formation of mesopores. From this research work, it was observed that the treatment at higher temperatures did not allow the formation of porosity in the hexagonal crystals of $\mathrm{ZnO}$. 
References:

[1] D. W. Bruce, D. O'Hare and R. I. Walton, "Porous materials", $1^{\text {st }}$ ed. (Wiley, United Kingdom, 2010) et al., p.1.

[2] L. H. Cheng et al., Elsevier vol. 38 (2012), pp S457-S461.

[3] J. N. Hasnidawani et al., Elsevier vol. 19 (2016), pp 211-216.

[4] Y. L. Zhang et al., Springer vol. 51 (2009), pp 198-203.

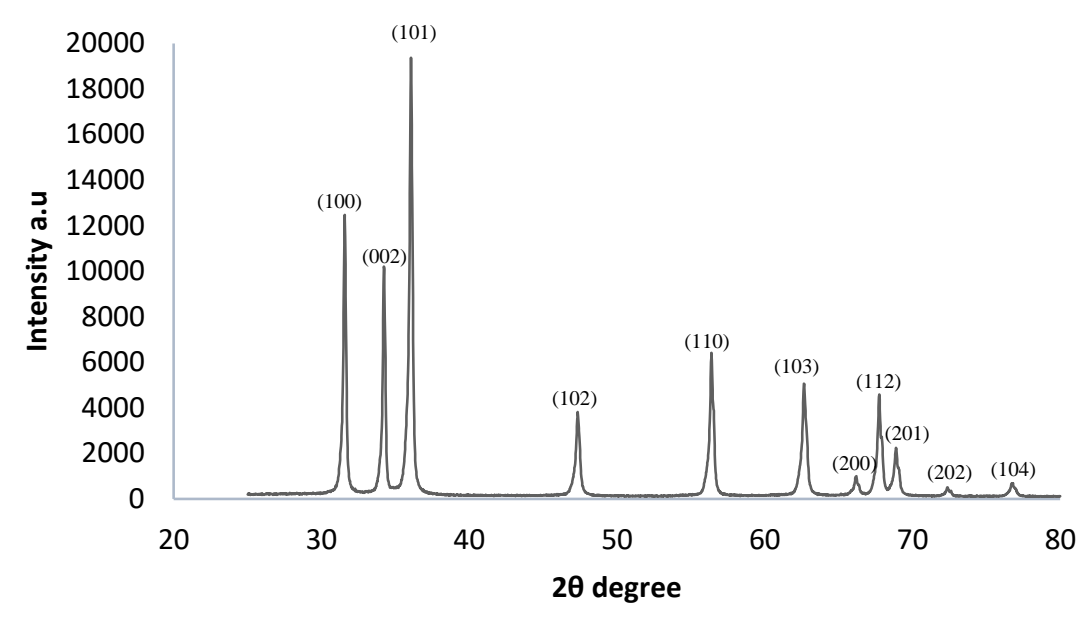

Figure 1. X-ray analysis of mesoporous zinc oxide particles.
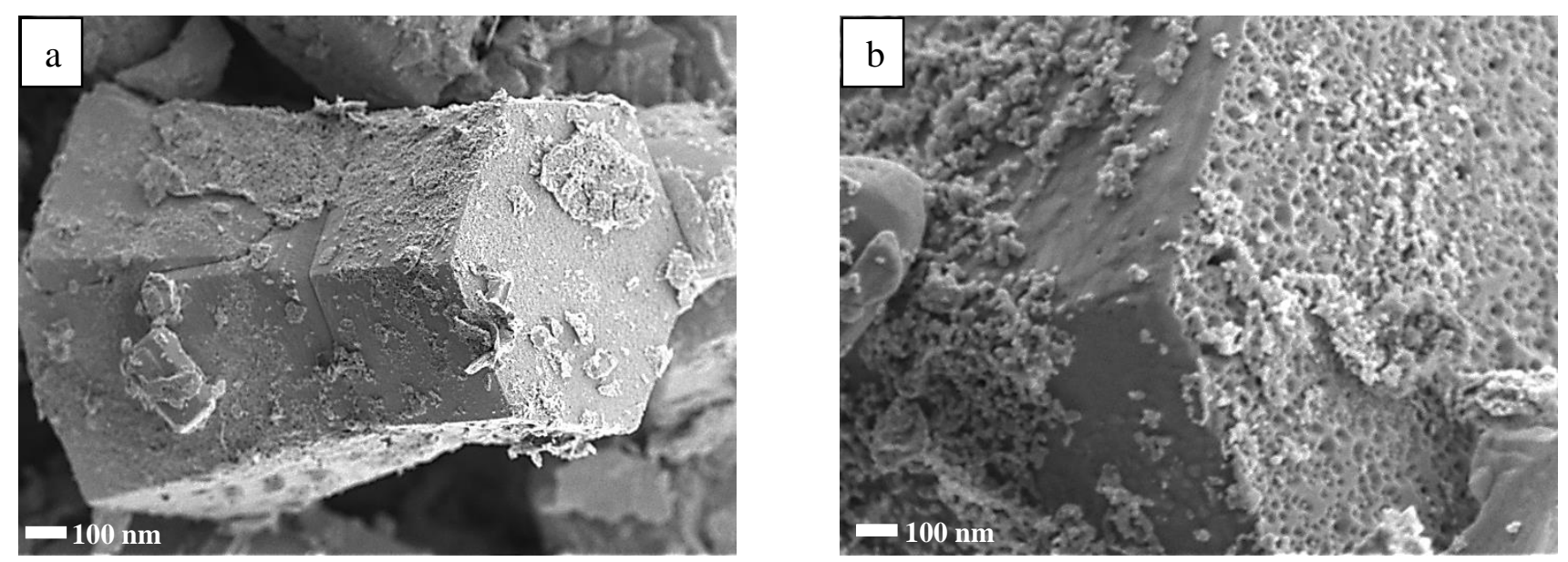

Figure 2. SEM images of zinc oxide sample after calcination at a) $5000 \mathrm{x}$ (hexagonal crystals of $\mathrm{ZnO}$ ), and b) $40000 \mathrm{x}$ (Mesopores) of magnification. 